\title{
A Fuzzy Reliability Model of Blades to Avoid Resonance and Its Convergence Analysis
}

\author{
Meng Zhang, Shan Lu, and Bo Li \\ School of Power and Energy, Northwestern Polytechnical University, Xian 710072, China \\ Correspondence should be addressed to Meng Zhang; nwpumeng@mail.nwpu.edu.cn
}

Received 22 January 2014; Revised 19 July 2014; Accepted 28 July 2014; Published 14 August 2014

Academic Editor: Thomas Hanne

Copyright (C) 2014 Meng Zhang et al. This is an open access article distributed under the Creative Commons Attribution License, which permits unrestricted use, distribution, and reproduction in any medium, provided the original work is properly cited.

\begin{abstract}
The inherent frequencies of blades, especially with friction structure of shroud, are related to many uncertain factors, which include not only random factors but also fuzzy or other uncertain factors. In this paper, the fuzzy reliability model of blades to avoid resonance is investigated. By regarding the inherent frequency as a fuzzy random variable with trapezoidal or flat-normal membership function, a fuzzy reliability model of blades to avoid resonance is proposed based on the fuzzy cut-set theory, and the corresponding numerical solution is given. Further, a theorem is proposed and proved to indicate the deficiency of the previous cut-set distributions on convergence, and, then, a new cut-set distribution is given to cover this shortage and ensure that the model is of good convergence. Finally, the proposed model is applied to evaluate the reliability of one certain blade. Some simulations are carried out to compare the new cut-set distribution with the previous ones and study the influence of fuzzy degree on the reliability.
\end{abstract}

\section{Introduction}

Blade is one of the important components of aeroengine, whose failure rate is always higher than other components due to its complex service environment. The failure of blades is often related to vibration, in which resonance failure accounts for $30 \% \sim 40 \%$ [1]. Therefore, it is of great significance to study the reliabilities of blades to avoid resonance.

The essence of the conventional random reliability theory is to regard the basic variables as random variables. However, in the process of vibration, the inherent frequencies of blades have not only randomness but also great fuzziness. The fuzziness is mainly due to the following. (1) The inherent frequencies of blades (especially with damping structures, such as shrouded blades) are affected by many factors, some of which have complex influence on inherent frequencies (e.g., positive pressure on contact surface has a high nonlinear effect [2]), some factors (such as circumferential position excursion of shroud to firtree serration and installation tightness between tenon and mortise) affect it irregularly, and even some unknown factors also might affect the inherent frequencies. Because of the multiple and composite influences, the inherent frequencies of blades with damping structures are of great fuzziness. (2) In the acquisition (test or simulation) process of the inherent frequencies, many steps depend on the experiences and subjective consciousness of engineers, such as the design of experiment scheme, experiment equipment, algorithm, and program. Experience and consciousness are both fuzzy concepts. These indicate that the fuzziness of inherent frequencies is inevitable and cannot be neglected. Just as the inherent frequencies of blades, the variables which include both fuzziness and randomness are called fuzzy random variables and should be handled by the fuzzy reliability theory in the reliability analysis.

The fuzzy reliability theory is based on the fuzzy set theory, which was proposed by Zadeh in 1965 [3] and had been applied in many fields [4-9]. The fuzziness in reliability problems mainly involves the fuzziness of failure state and the fuzziness of basic variables. According to the type of fuzziness in the model, the fuzzy reliability models could be classified into three groups [10-12]: the model which is only based on the fuzziness of failure state is called Profust model, the model which is only based on the fuzziness of basic variables is called Posbist model, and the model which is based on the fuzziness of both failure state and basic variables is called Posfust model. At present, the studies on the fuzzy reliability to avoid resonance are mostly the Profust models, which ignore the fuzziness of basic variable (inherent frequency) 
[13-16]. Therefore, this paper is aimed to study the Posbist reliability model of blades to avoid resonance.

To deal with fuzzy random variables, some methods in the existing studies (such as "stress-strength" interference model with the fuzziness of stress or strength [17-20]) are worth learning. The basic idea is to translate the fuzzy random variables into pure random variables by some defuzzification methods, and then the mature random theories could be used. The cut-set method is one of the common defuzzification methods, in which it is very important to choose an appropriate cut-set distribution due to its significant effects on the analysis results. At present, the common cut-set distributions include uniform distribution, linear distribution, and truncated normal distribution [21-23]. However, when the cut-set is longer, these distributions become unreasonable and cannot reflect the random characteristics of variables. This deficiency will bring trouble to the convergence of the model, and thus a new cut-set distribution will be proposed in this paper to cover the shortage of the previous cut-set distributions.

This paper is organized as follow. In Section 2, two kinds of membership functions were adopted to describe the fuzziness of the inherent frequency, and their fuzzy degrees were discussed. In Section 3, a Posbist reliability model of blades to avoid resonance was proposed based on the fuzzy cut-set theory, and a numerical solution was given. In Section 4, the convergence of the Posbist model was analyzed. A convergence theorem was given and proved to indicate the deficiency of the previous cut-set distributions, and a new distribution was proposed to ensure the convergence of the Posbist model. And in Section 5, simulations were carried out in order to verify the theoretical results and compare the new cut-set distribution with the previous ones, and the influences of fuzzy degree on the reliability were investigated. Some conclusions were made in Section 6.

\section{Description and Measurement of the Fuzzy Random Inherent Frequency}

The fuzziness of fuzzy random variable can be described by a membership function $\mu_{\widetilde{A}}(u) \in[0,1]$, where $\widetilde{A}$ is a set in the domain $U$ and $\widetilde{A}=\{$ the possible values of this variable $\}$. For some $u \in U$, the value $\mu_{\widetilde{A}}(u)$ represents the degree of event $u \in \widetilde{A}$ and is called membership degree. Therefore, $\mu_{\widetilde{A}}(u)=$ 0 represents $u \notin \widetilde{A}, \mu_{\widetilde{A}}(u)=1$ represents $u \in \widetilde{A}$, and $0<$ $\mu_{\widetilde{A}}(u)<1$ represents whether $u$ belongs to $\widetilde{A}$ is uncertain, and the possibility is $\mu_{\widetilde{A}}(u)$. These show that the elements in $\widetilde{A}$ are fuzzy and uncertain, so $\widetilde{A}$ is not an ordinary set but a fuzzy set.

In engineering, the membership function $\mu_{\widetilde{A}}(u)$ of a fuzzy random variable generally satisfies the following format:

$$
\mu_{\widetilde{A}}(u)= \begin{cases}L(u) & a-M_{1} \leq u<a \\ 1 & a \leq u \leq b \\ R(u) & b<u \leq b+M_{2} \\ 0 & \text { else, }\end{cases}
$$

where $[a, b]$ is called flat zone, $0 \leq L(u)<1$ is an increasing left continuous function, and $\lim _{u \rightarrow-\infty} L(u)=0$; $0 \leq R(u)<1$ is a decreasing right continuous function and $\lim _{u \rightarrow+\infty} R(u)=0$. The linear or normal function is usually chosen as $L(u)$ and $R(u)$ in engineering.

2.1. Membership Function of Inherent Frequency. The inherent frequency is a fuzzy random variable, so the set $\tilde{f}_{n}=\{$ the possible values of the inherent frequency\} is a fuzzy set and could be described by the membership function $\mu_{\widetilde{f}_{n}}\left(f_{n}\right)$. $\mu_{\tilde{f}_{n}}\left(f_{n}\right)$ can be determined through the expression (1). Generally speaking, the inherent frequency of one blade is designed to be some value, but the dispersion is inevitable due to the manufacture dispersion. A certain frequency tolerance is allowed in engineering. In the predetermined tolerance range, the inherent frequency can be deemed to be clear. So the tolerance interval is just the flat zone, where the membership degree is the constant 1. Practically, the ultratolerance phenomenon sometimes may occur; that is to say, the inherent frequency is fuzzy within a certain range on both sides of the flat zone, where the membership degree is determined by functions $L\left(f_{n}\right)$ and $R\left(f_{n}\right)$. If $L\left(f_{n}\right)$ and $R\left(f_{n}\right)$ are linear functions, the membership function is trapezoidal (expression (2)), and if they are normal functions, the membership function is flat-normal (expression (3)).

(I) Trapezoidal membership function is as follows:

$$
\mu_{\tilde{f}_{n}}\left(f_{n}\right)= \begin{cases}1-\frac{a-f_{n}}{M_{1}} & a-M_{1} \leq f_{n}<a \\ 1 & a \leq f_{n} \leq b \\ 1-\frac{f_{n}-b}{M_{2}} & b<f_{n} \leq b+M_{2} \\ 0 & \text { else, }\end{cases}
$$

where $\left(a-M_{1}, a\right)$ and $\left(b, b+M_{2}\right)$ are fuzzy zones and $M_{1}$ and $M_{2}$ are fuzzy-information parameters and are used to characterize the length of fuzzy zones.

(II) Flat-normal membership function is as follows:

$$
\mu_{\widetilde{f}_{n}}\left(f_{n}\right)= \begin{cases}\exp \left\{-K_{1}\left(f_{n}-a\right)^{2}\right\} & f_{n}<a \\ 1 & a \leq f_{n} \leq b \\ \exp \left\{-K_{2}\left(f_{n}-b\right)^{2}\right\} & f_{n}>b,\end{cases}
$$

where $(-\infty, a)$ and $(b,+\infty)$ are fuzzy zones and $K_{1}$ and $K_{2}$ are fuzzy-information parameters and are used to adjust the shape of membership functions.

If there is no additional information, it should be taken that $M_{1}=M_{2}\left(K_{1}=K_{2}\right)$. In practice, for a specific blade, the membership function of its inherent frequency can be chosen as the specific one from (2) and (3) by some methods, such as statistics, function fitting, or the experience of experts.

2.2. Fuzzy Degree of the Inherent Frequency. Fuzzy degree is used to measure the ambiguity of a fuzzy random variable through its membership function. In continuous domain, 
fuzzy degree can be calculated according to the following expression [24]:

$$
D(\mu)=\int_{-\infty}^{+\infty}\left|\mu(x)-\mu_{0.5}(x)\right| d x,
$$

where $\mu_{0.5}(x)=\left\{\begin{array}{l}1 \mu(x) \geq 0.5 \\ 0 \mu(x)<0.5\end{array}\right.$. The fuzzy degrees of trapezoidal and flat-normal fuzzy random variables can be calculated according to the expression (4), and the results are shown as follows.

(I) The fuzzy degree of trapezoidal membership function is as follows:

$$
\begin{aligned}
D(\mu)= & \int_{-\infty}^{a-\left(M_{1} / 2\right)} \mu_{\tilde{f}_{n}}\left(f_{n}\right) d f_{n}+\int_{\left(a-M_{1}\right) / 2}^{\left(b+M_{2}\right) / 2}\left(1-\mu_{\tilde{f}_{n}}\left(f_{n}\right)\right) d f_{n} \\
& +\int_{\left(b+M_{2}\right) / 2}^{+\infty} \mu_{\tilde{f}_{n}}\left(f_{n}\right) d f_{n}=\frac{1}{4}\left(M_{1}+M_{2}\right) .
\end{aligned}
$$

(II) The fuzzy degree of Flat-normal membership function is as follows:

$$
\begin{aligned}
D(\mu)= & \int_{-\infty}^{a-\left(\sqrt{\ln 2} / \sqrt{K_{1}}\right)} \mu_{\tilde{f}_{n}}\left(f_{n}\right) d f_{n} \\
& +\int_{a-\left(\sqrt{\ln 2} / \sqrt{K_{1}}\right)}^{b+\left(\sqrt{\ln 2} / K_{2}\right)}\left(1-\mu_{\tilde{f}_{n}}\left(f_{n}\right)\right) d f_{n} \\
& +\int_{b+\left(\sqrt{\ln 2} / \sqrt{K_{2}}\right)}^{+\infty} \mu_{\tilde{f}_{n}}\left(f_{n}\right) d f_{n} \\
\approx & 0.3700\left(\frac{1}{\sqrt{K_{1}}}+\frac{1}{\sqrt{K_{2}}}\right) .
\end{aligned}
$$

In summary, the fuzzy degree of a fuzzy random variable only relates to the fuzzy-information parameters of its membership function and has nothing to do with its precise information, that is, the flat zone $[a, b]$.

Particularly, when $M_{1}=M_{2} \rightarrow+\infty\left(\right.$ or $\left.K_{1}=K_{2}=0\right)$, the membership function (2) (or (3)) will degenerate into the constant function:

$$
\mu_{\tilde{f}_{n}}\left(f_{n}\right) \equiv 1 ; \quad f_{n} \in(-\infty,+\infty) .
$$

In this case, the set $\widetilde{f}_{n}$ degenerates into ordinary set and the fuzzy degree of the inherent frequency will be 0 according to the expression (4), which indicates that the inherent frequency is no longer a fuzzy random variable but a pure random variable.

\section{Posbist Reliability Model of Blades to Avoid Resonance}

Suppose that the inherent frequency $\tilde{f}_{n}$ of one blade is a fuzzy random variable, whose membership function is $\mu_{\tilde{f}_{n}}\left(f_{n}\right)$. The excitation frequency is a random variable and follows normal distribution $N\left(\mu_{e}, \sigma_{e}^{2}\right)$, whose density function is $g_{f_{e}}\left(f_{e}\right)$.
3.1. The Posbist Reliability Model. The reliability of this blade to avoid resonance is shown as follows:

$$
\begin{aligned}
R & =P\left(\left|\tilde{f}_{n}-f_{e}\right|>\alpha\right) \\
& =P\left(\tilde{f}_{n}-f_{e}+\alpha<0\right)+P\left(f_{e}-\tilde{f}_{n}+\alpha<0\right) \\
& \triangleq R_{1}+R_{2} .
\end{aligned}
$$

Define $f_{e^{\prime}}=f_{e}-\alpha$ and $f_{e^{\prime \prime}}=f_{e}+\alpha$; thus there are $f_{e^{\prime}} \sim N\left(\mu_{e}-\alpha, \sigma_{e}^{2}\right)$ and $f_{e^{\prime \prime}} \sim N\left(\mu_{e}+\alpha, \sigma_{e}^{2}\right)$. Suppose that the density function and survival function of $f_{e^{\prime}}$ are $g_{f_{e^{\prime}}}\left(f_{e^{\prime}}\right)$ and $F_{f_{e^{\prime}}}\left(f_{e^{\prime}}\right)$, respectively and those of $f_{e^{\prime \prime}}$ are $g_{f_{e^{\prime \prime}}}\left(f_{e^{\prime \prime}}\right)$ and $F_{f_{e^{\prime \prime}}}\left(f_{e^{\prime \prime}}\right)$. Then,

$$
R_{1}=P\left(\tilde{f}_{n}<f_{e^{\prime}}\right), \quad R_{2}=P\left(\tilde{f}_{n}>f_{e^{\prime \prime}}\right) .
$$

The reliability to avoid resonance is studied based on the fuzzy cut-set theory. A cut level $\lambda \in[0,1]$ is given first, and, then, the $\lambda$ cut-set of $\tilde{f}_{n}$ can be calculated as $\left[a_{\lambda}, b_{\lambda}\right]=$ $\left\{f_{n}: \mu_{\tilde{f}_{n}}\left(f_{n}\right) \geq \lambda\right\}$, which is an ordinary set. By introducing a random density function $g_{f_{n}, \lambda}\left(f_{n}\right)$ into the cut-set $\left[a_{\lambda}, b_{\lambda}\right]$, the cut-set reliability $R_{\lambda}=R_{1 \lambda}+R_{2 \lambda}$ can be obtained based on the conventional random reliability theory, where

$$
\begin{aligned}
R_{1 \lambda} & =\int_{a_{\lambda}}^{b_{\lambda}} g_{f_{n}, \lambda}\left(f_{n}\right)\left[\int_{f_{n}}^{+\infty} g_{f_{e^{\prime}}}\left(f_{e^{\prime}}\right) d f_{e^{\prime}}\right] d f_{n} \\
& =1-\int_{a_{\lambda}}^{b_{\lambda}} g_{f_{n}, \lambda}\left(f_{n}\right) F_{f_{e^{\prime}}}\left(f_{n}\right) d f_{n}, \\
R_{2 \lambda} & =\int_{a_{\lambda}}^{b_{\lambda}} g_{f_{n}, \lambda}\left(f_{n}\right)\left[\int_{-\infty}^{f_{n}} g_{f_{e^{\prime \prime}}}\left(f_{e^{\prime \prime}}\right) d f_{e^{\prime \prime}}\right] d f_{n} \\
& =\int_{a_{\lambda}}^{b_{\lambda}} g_{f_{n}, \lambda}\left(f_{n}\right) F_{f_{e^{\prime \prime}}}\left(f_{n}\right) d f_{n^{\prime}} .
\end{aligned}
$$

Because $g_{f_{n}, \lambda}\left(f_{n}\right)$ is the random density function and defined in the cut-set $\left[a_{\lambda}, b_{\lambda}\right]$, it is called cut-set distribution. Taking the cut level $\lambda$ as the integral variable and integrating $R_{\lambda}$ into the interval $[0,1]$, the Posbist fuzzy reliability model of blades to avoid resonance can be proposed as follows:

$$
R=\int_{0}^{1} R_{\lambda} d \lambda=\int_{0}^{1}\left(R_{1 \lambda}+R_{2 \lambda}\right) d \lambda
$$

3.2. Calculation of the Model. According to the formulas in (10), after a group of $f_{n k \mid \lambda}\left(k=1,2, \ldots, N_{n}\right)$ are simulated randomly in the cut-set $\left[a_{\lambda}, b_{\lambda}\right], R_{\lambda}$ can be calculated by the two formulas below:

$$
\begin{aligned}
R_{1 \lambda} & =1-E_{f_{n} \mid \lambda}\left[\Phi\left(\frac{f_{n}-\mu_{e^{\prime}}}{\sigma_{e^{\prime}}}\right)\right] \\
& \approx 1-\frac{1}{N_{n}} \sum_{k=1}^{N_{n}} \Phi\left(\frac{f_{n k \mid \lambda}-\mu_{e^{\prime}}}{\sigma_{e^{\prime}}}\right),
\end{aligned}
$$

$$
R_{2 \lambda}=E_{f_{n} \mid \lambda}\left[\Phi\left(\frac{f_{n}-\mu_{e^{\prime \prime}}}{\sigma_{e^{\prime \prime}}}\right)\right] \approx \frac{1}{N_{n}} \sum_{k=1}^{N_{n}} \Phi\left(\frac{f_{n k \mid \lambda}-\mu_{e^{\prime \prime}}}{\sigma_{e^{\prime \prime}}}\right) .
$$




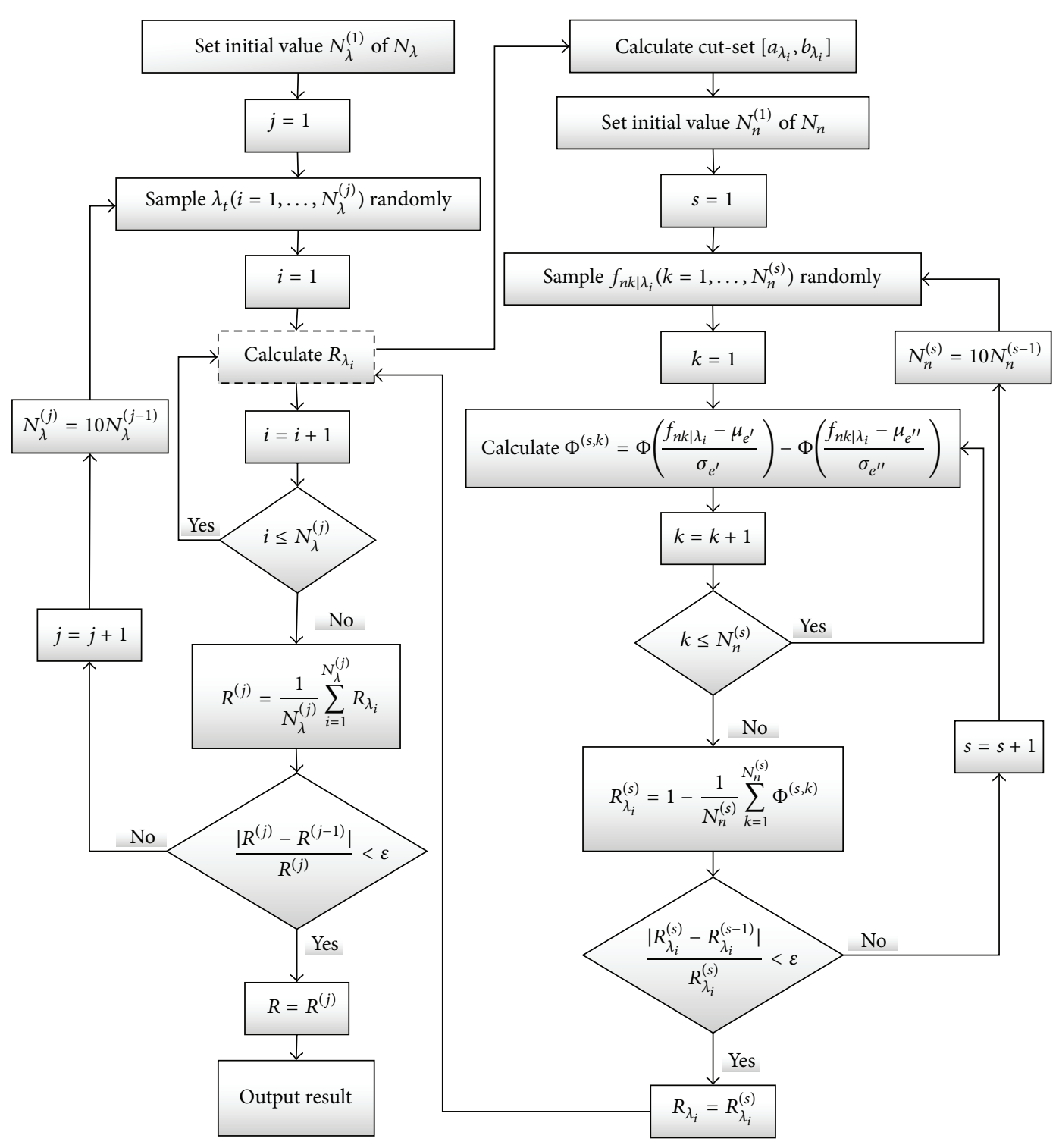

FIgURE 1: Flowchart of the numerical simulation method.

The cut level $\lambda$ is regarded as a random variable which follows uniform distribution in [0,1], and the formula (11) can be written as $R=\int_{0}^{1} R_{\lambda} d \lambda=E_{\lambda}\left[R_{\lambda}\right]$. Therefore, a group of cut levels $\lambda_{i}\left(i=1,2, \ldots, N_{\lambda}\right)$ can be simulated randomly, and the corresponding cut-set reliabilities $R_{\lambda_{i}}$ can be calculated according the formulas in (12). Then, the fuzzy reliability $R$ to avoid resonance can be calculated as

$$
R \approx \frac{1}{N_{\lambda}} \sum_{i=1}^{N_{\lambda}} R_{\lambda_{i}}
$$

The calculation results are related to the sample sizes $N_{\lambda}$ and $N_{n}$, and thus an iterative method can be used. The flowchart of the numerical simulation method is shown in Figure 1.

\section{Convergence of the Posbist Model and a New Cut-Set Distribution}

According to the model in (11), the cut-set distribution $g_{f_{n}, \lambda}\left(f_{n}\right)$ introduced into the cut-set $\left[a_{\lambda}, b_{\lambda}\right]$ is of significant effect on the reliability, and, thus, it is very important to choose an ideal cut-set distribution. At present, the common cut-set distributions are uniform distribution (UD), linear distribution (LD), and truncated normal distribution (TND). In order to point out the deficiency of these previous cut-set distributions, the convergence of the Posbist model should be analyzed first.

4.1. Convergence of the Posbist Model. As is well known, the pure random variable is a special fuzzy random variable, which no longer has fuzziness. When the fuzzy random inherent frequency gradually converges to a pure random 
variable, the Posbist model should converge to the corresponding random model. Particularly, when the membership function of the inherent frequency degenerates into the constant function $\mu_{\tilde{f}_{n}}\left(f_{n}\right) \equiv 1, f_{n} \in(-\infty,+\infty)$, the inherent frequency should degenerate into a random variable which obeys the probability function $g_{f_{n}}\left(f_{n}\right)$ in $(-\infty,+\infty)$. In this case, according to the Posbist model, it can be seen that the cut-sets corresponding to arbitrary cut levels are all $(-\infty,+\infty)$. Thus, $g_{f_{n}, \lambda}\left(f_{n}\right)$ has nothing to do with $\lambda$ and can be denoted by $g_{f_{n}}\left(f_{n}\right)$. Therefore, the cut-set reliabilities to arbitrary cut levels are equal and have no relationship to $\lambda$ and can be denoted by $R^{\prime}$. Through the expression (11), it is obtained that $R=R^{\prime}$. Because $R^{\prime}$ is the random reliability model where the inherent frequency follows the random density function $g_{f_{n}}\left(f_{n}\right)$ in $(-\infty,+\infty)$, the Posbist model degenerates into a random model.

In fact, $g_{f_{n}}\left(f_{n}\right)$ should reflect the random characteristic of the inherent frequency. Therefore, it should just be the probability density function of random inherent frequency in conventional random model. In this way, the conventional random model is a special Posbist model, while the Posbist method is the development and extension of the random reliability method. Because $g_{f_{n}}\left(f_{n}\right)$ is the limit function of $g_{f_{n}, \lambda}\left(f_{n}\right)$ when the cut-set tends to $(-\infty,+\infty)$, an ideal cutset distribution $g_{f_{n}, \lambda}\left(f_{n}\right)$ should well reflect the random characteristic of the inherent frequency and converge to the probability density function of the inherent frequency in the conventional random model.

4.2. Deficiency of the Previous Cut-Set Distributions. At present, the common $g_{f_{n}, \lambda}\left(f_{n}\right)$ is UD, LD, or TND. These cutset distributions can reflect the random characteristic of the inherent frequency when the cut-set $\left[a_{\lambda}, b_{\lambda}\right]$ is short. But if the cut-set $\left[a_{\lambda}, b_{\lambda}\right]$ is longer and tends, especially, to $(-\infty,+\infty)$, $\mathrm{LD}$ and TND tend to UD, which means that the inherent frequency takes equal probability everywhere in $(-\infty,+\infty)$ (Figure 2). This is unreasonable and brings a deficiency to the convergence of the Posbist model, which is shown in the following lemma and theorem.

Lemma 1. If the cut-set distribution is UD, LD, or TND, when the $\lambda$ cut-set of the inherent frequency converges to real field, that is, $\left[a_{\lambda}, b_{\lambda}\right] \rightarrow(-\infty,+\infty)$, the cut-set reliability $R_{\lambda}$ to avoid resonance will converge to the fixed value 1 and has nothing to do with the specific issues.

Proof. When $\left[a_{\lambda}, b_{\lambda}\right] \rightarrow(-\infty,+\infty)$, the cut-set distributions $\mathrm{LD}$ and TND will converge to UD. Thus, it is just needed to prove UD.

For arbitrary fixed $f_{e^{\prime}}, f_{e^{\prime \prime}} \in(-\infty,+\infty)$, when $\left[a_{\lambda}, b_{\lambda}\right]$ is long enough and tends to $(-\infty,+\infty)$, there is always $f_{e^{\prime}}, f_{e^{\prime \prime}} \in$ $\left[a_{\lambda}, b_{\lambda}\right]$. Therefore, according to the formulas in (10), $R_{1 \lambda}$ and $R_{2 \lambda}$ can be written in the equivalent forms below:

$$
\begin{aligned}
& R_{1 \lambda}=\int_{-\infty}^{+\infty} g_{f_{e^{\prime}}}\left(f_{e^{\prime}}\right)\left[\int_{a_{\lambda}}^{f_{e^{\prime}}} g_{f_{n}, \lambda}\left(f_{n}\right) d f_{n}\right] d f_{e^{\prime}}, \\
& R_{2 \lambda}=\int_{-\infty}^{+\infty} g_{f_{e^{\prime \prime}}}\left(f_{e^{\prime \prime}}\right)\left[\int_{f_{e^{\prime \prime}}}^{b_{\lambda}} g_{f_{n}, \lambda}\left(f_{n}\right) d f_{n}\right] d f_{e^{\prime \prime}}
\end{aligned}
$$

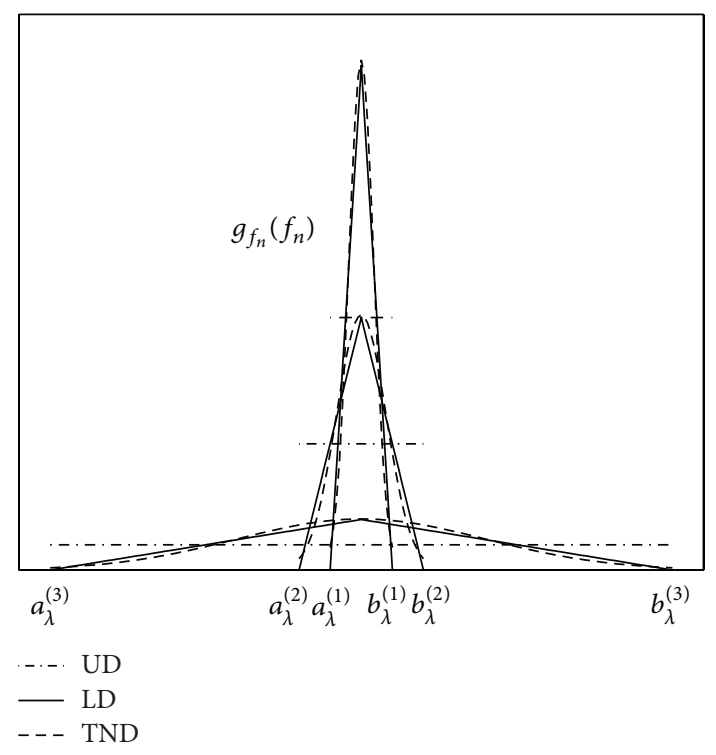

FIgURE 2: UD, LD, and TND in the different cut-sets.

When the cut-set distribution $g_{f_{n}, \lambda}\left(f_{n}\right)$ is UD, substitute it into the above formulas and the following expression can be obtained:

$$
\begin{aligned}
R_{\lambda}= & R_{1 \lambda}+R_{2 \lambda}=\int_{-\infty}^{+\infty} g_{f_{e^{\prime}}}\left(f_{e^{\prime}}\right)\left[\frac{f_{e^{\prime}}-a_{\lambda}}{b_{\lambda}-a_{\lambda}}\right] d f_{e^{\prime}} \\
& +\int_{-\infty}^{+\infty} g_{f_{e^{\prime \prime}}}\left(f_{e^{\prime \prime}}\right)\left[\frac{b_{\lambda}-f_{e^{\prime \prime}}}{b_{\lambda}-a_{\lambda}}\right] d f_{e^{\prime \prime}} \\
= & \frac{E\left[f_{e^{\prime}}\right]-a_{\lambda}}{b_{\lambda}-a_{\lambda}}+\frac{b_{\lambda}-E\left[f_{e^{\prime \prime}}\right]}{b_{\lambda}-a_{\lambda}} \\
= & 1-\frac{2 \alpha}{b_{\lambda}-a_{\lambda}} \longrightarrow 1
\end{aligned}
$$

In specific engineering problems, different blades will lead different parameters in the Posbist model. However, the above analysis shows that no matter what values of these parameters are, the cut-set reliability $R_{\lambda}$ will always converge to 1 when $\left[a_{\lambda}, b_{\lambda}\right] \rightarrow(-\infty,+\infty)$. In other words, it has nothing to do with the specific issues.

According the lemma above, the following theorem can be derived.

Theorem 2 (convergence theorem of the Posbist model under the cut-set distribution $\mathrm{UD}, \mathrm{LD}$, or TND). If the cut-set distribution is $U D, L D$, or TND, when the membership function of the inherent frequency gradually converges to $\mu_{\tilde{f}_{n}}\left(f_{n}\right) \equiv 1$, $f_{n} \in(-\infty,+\infty)$, the reliability assessment to avoid resonance using the Posbist model will converge to 1 and has nothing to do with the specific issues.

Proof. When the membership function converges to $\mu_{\tilde{f}_{n}}\left(f_{n}\right) \equiv 1, f_{n} \in(-\infty,+\infty)$, for an arbitrary fixed $\lambda \in[0,1]$, the corresponding cut-set $\left[a_{\lambda}, b_{\lambda}\right]=\left\{f_{n}: \mu_{\tilde{f}_{n}}\left(f_{n}\right) \geq \lambda\right\}$ 


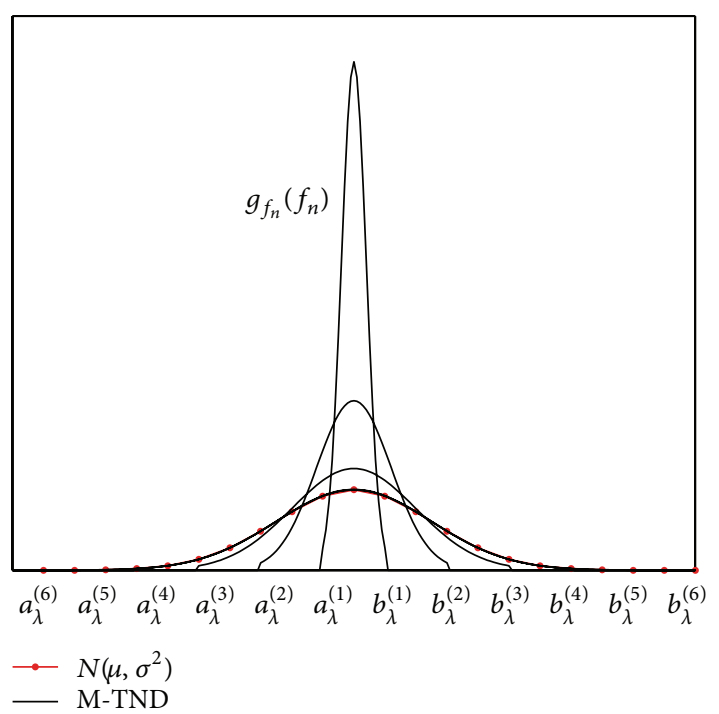

Figure 3: M-TND in the different cut-sets.

always converges to $(-\infty,+\infty)$. According to the lemma above, if the cut-set distribution is UD, LD, or TND, the corresponding cut-set reliability $R_{\lambda} \rightarrow 1$. Thus, based on the formula (11), it can be obtained that $R=\int_{0}^{1} R_{\lambda} d \lambda \rightarrow 1$ and will have nothing to do with the specific problem.

The analysis in Section 4.1 shows that an ideal cut-set distribution should ensure that the Posbist model converges to the conventional random model when $\mu_{\tilde{f}_{n}}\left(f_{n}\right) \equiv 1, f_{n} \in$ $(-\infty,+\infty)$. However, the above theorem shows that UD, LD, and TND will make the Posbist reliability assessments converge to 1 invariably, which is obviously unreasonable. The essence for this is because UD, LD, and TND cannot reflect the random characteristic of the inherent frequency when the cut-set is long enough, and, thus, a new cut-set distribution should be proposed.

4.3. A New Cut-Set Distribution. As the analysis in Section 4.1, $g_{f_{n}, \lambda}\left(f_{n}\right)$ should converge to $g_{f_{n}}\left(f_{n}\right)$ when $\left[a_{\lambda}, b_{\lambda}\right] \rightarrow(-\infty,+\infty) \cdot g_{f_{n}}\left(f_{n}\right)$ is just the probability density function of the inherent frequency in the conventional random method, and it always is assumed as a normal distribution. Therefore, a new cut-set distribution called modified truncated normal distribution (M-TND) is proposed in this paper, whose expression is shown as follows:

$$
g_{f_{n}, \lambda}\left(f_{n}\right)= \begin{cases}\frac{f\left(f_{n}\right)}{\int_{a_{\lambda}}^{b_{\lambda}} f\left(f_{n}\right) d f_{n}} & a_{\lambda} \leq f_{n} \leq b_{\lambda} \\ 0 & \text { else, }\end{cases}
$$

where $f\left(f_{n}\right)=(1 / \sqrt{2 \pi} \bar{\sigma}) \exp \left\{-\left(f_{n}-\mu\right)^{2} / 2 \bar{\sigma}^{2}\right\}, \mu=\left(a_{\lambda}+\right.$ $\left.b_{\lambda}\right) / 2$, and $\bar{\sigma}=\min \left\{\sigma,\left(b_{\lambda}-a_{\lambda}\right) / C\right\}$. C is the standard deviation coefficient. $\sigma$ is given by the expertise and generally takes the standard deviation of the inherent frequency in the random reliability model.

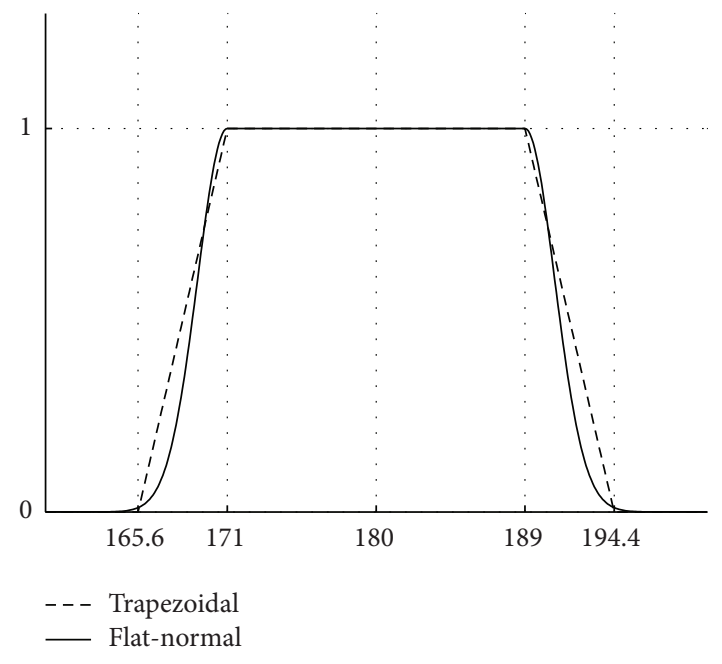

FIGURE 4: Membership functions of the inherent frequency.

According to (16), M-TND will converge to normal distribution $N\left(\mu, \sigma^{2}\right)$ when $\left[a_{\lambda}, b_{\lambda}\right] \rightarrow(-\infty,+\infty)$ (Figure 3$)$, and, therefore, the reliability assessment of model (11) will converge to the result of the random model.

\section{Simulation Study}

The first bend frequency of a known shroud blade is designed to be about $180 \mathrm{~Hz}$. The manufacture tolerance is $\pm 5 \%$. While the ultratolerance phenomenon may appear in practice and the acceptable ultratolerance range is $\pm 8 \%$ in engineering. When the blade is shocked by the excitation frequency which follows random distribution $N\left(220,7^{2}\right)$, the resonance may occur. Now the reliability to avoid resonance will be estimated.

From the known conditions, the median and length of the flat zone of the first bend frequency are 180 and 18, respectively; that is, $[a, b]=[171,189]$. The trapezoidal and flat-normal membership functions are considered. For the former, the lengths of left and right fuzzy zones are both $L_{f}=180 \times(8 \%-5 \%)=5.4$. So the value can be taken as $M_{1}=M_{2} \triangleq M=5.4$. For the latter, $K_{1}=K_{2} \triangleq$ $K=\left(L_{f} / 3\right)^{-2} / 2=0.1543$ [25]. The two membership functions of the inherent frequency are shown in Figure 4. Reliabilities to avoid resonance can be calculated as 0.9926 and 0.9929 , respectively, for these two membership functions by the Posbist model proposed.

5.1. Comparison of M-TND with the Previous Cut-Set Distributions (UD, LD, and TND). In order to study the convergence of the Posbist model under different cut-set distributions, the reliability variations with the fuzzy-information parameters $M$ and $K$ are calculated and shown in Tables 1 and 2 and Figures 5 and 6 . It is shown in Tables 1 and 2 that when the membership function of the inherent frequency converges to $\mu_{\tilde{f}_{n}}\left(f_{n}\right) \equiv 1, f_{n} \in(-\infty,+\infty)(M \rightarrow+\infty$ or $K \rightarrow$ 0 ), the reliability assessments under UD, LD, and TND always converge to 1 , which is consistent with the theorem 
TABLE 1: The reliability assessments under UD, LD, and TND with the change of $M$.

\begin{tabular}{lccccccccc}
\hline$M$ & 0 & 50 & $10^{2}$ & $10^{3}$ & $10^{4}$ & $10^{5}$ & $10^{6}$ & $10^{7}$ \\
\hline UD & 0.9903 & 0.8150 & 0.7802 & 0.9323 & 0.9899 & 0.9982 & 0.9998 & 1.0000 & 1.0000 \\
LD & 0.9945 & 0.8901 & 0.8296 & 0.9063 & 0.9817 & 0.9980 & 0.9996 & 1.0000 & 1.0000 \\
TND & & & & & & & & \\
$\quad C=3$ & 0.9925 & 0.8565 & 0.8044 & 0.9202 & 0.9849 & 0.9983 & 0.9998 & 1.0000 & 1.0000 \\
$C=4$ & 0.9938 & 0.8793 & 0.8175 & 0.9110 & 0.9818 & 0.9975 & 0.9997 & 1.0000 & 1.0000 \\
$C=5$ & 0.9949 & 0.9068 & 0.8363 & 0.9014 & 0.9807 & 0.9969 & 0.9997 & 1.0000 & 1.0000 \\
$C=6$ & 0.9958 & 0.9256 & 0.8548 & 0.8889 & 0.9781 & 0.9973 & 0.9997 & 1.0000 & 1.0000 \\
$C=7$ & 0.9964 & 0.9445 & 0.8744 & 0.8826 & 0.9759 & 0.9968 & 0.9994 & 1.0000 & 1.0000 \\
$C=8$ & 0.9967 & 0.9591 & 0.8879 & 0.8723 & 0.9732 & 0.9953 & 0.9994 & 1.0000 & 1.0000 \\
\hline
\end{tabular}

TABLE 2: The reliability assessments under UD, LD, and TND with the change of $K$.

\begin{tabular}{lccccccccccccc}
\hline$K$ & $10^{-2}$ & $10^{-3}$ & $10^{-4}$ & $10^{-5}$ & $10^{-6}$ & $10^{-7}$ & $10^{-8}$ & $10^{-9}$ & $10^{-10}$ & $10^{-11}$ & $10^{-12}$ & $10^{-13}$ \\
\hline UD & 0.9412 & 0.8001 & 0.7957 & 0.9085 & 0.9679 & 0.9896 & 0.9966 & 0.9988 & 0.9996 & 0.9999 & 1.0000 & 1.0000 \\
LD & 0.9739 & 0.8835 & 0.7944 & 0.8639 & 0.9423 & 0.9799 & 0.9932 & 0.9978 & 0.9993 & 0.9998 & 0.9999 & 1.0000 \\
TND & & & & & & & & & & & \\
$C=3$ & 0.9587 & 0.8405 & 0.7900 & 0.8862 & 0.9561 & 0.9847 & 0.9953 & 0.9984 & 0.9995 & 0.9999 & 1.0000 & 1.0000 \\
$C=4$ & 0.9679 & 0.8690 & 0.7943 & 0.8701 & 0.9487 & 0.9827 & 0.9940 & 0.9981 & 0.9994 & 0.9998 & 0.9999 & 1.0000 \\
$C=5$ & 0.9767 & 0.8974 & 0.7964 & 0.8554 & 0.9388 & 0.9775 & 0.9928 & 0.9978 & 0.9992 & 0.9997 & 0.9999 & 1.0000 \\
$C=6$ & 0.9844 & 0.9189 & 0.8038 & 0.8403 & 0.9255 & 0.9761 & 0.9914 & 0.9974 & 0.9991 & 0.9997 & 0.9999 & 1.0000 \\
$C=7$ & 0.9884 & 0.9410 & 0.8148 & 0.8271 & 0.9202 & 0.9704 & 0.9906 & 0.9969 & 0.9989 & 0.9997 & 0.9999 & 1.0000 \\
$C=8$ & 0.9911 & 0.9513 & 0.8306 & 0.8191 & 0.9119 & 0.9654 & 0.9891 & 0.9963 & 0.9987 & 0.9996 & 0.9999 & 1.0000 \\
\hline
\end{tabular}

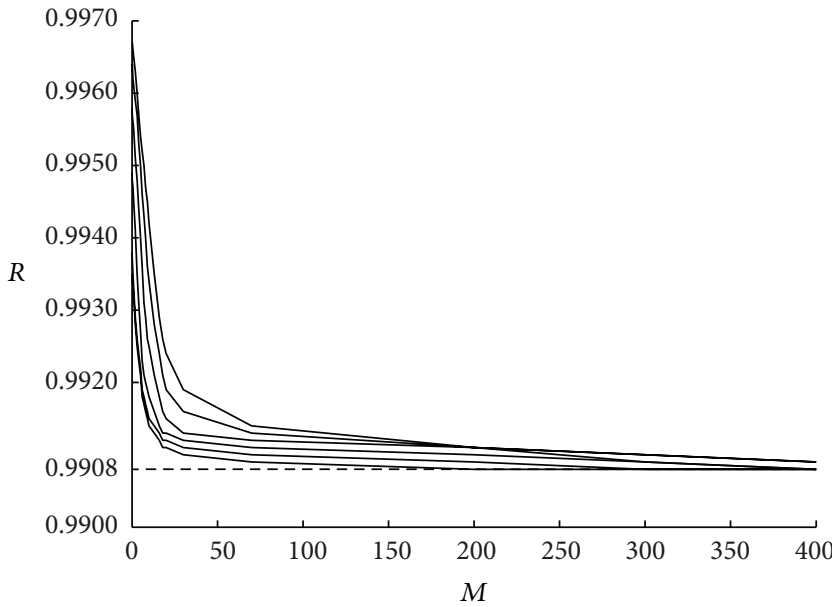

FIgure 5: Fuzzy reliabilities with $M$ for different $C$.

in Section 4.2. Meanwhile, it is shown in Figures 5 and 6 that the reliability assessments under M-TND converge to 0.9908 , which is just the result of the random reliability model.

In addition, it is also shown in Figures 5 and 6 that the reliability assessments under M-TND decreases with the increasing of $M$ and the decreasing of $K$. According to the formulas (5) and (6), when the fuzzy information flank is symmetrical, the higher the fuzzy degree of the inherent frequency is, the smaller the reliability will be and vice versa. This is consistent with the objective reality. However, as shown in Tables 1 and 2, with the increasing of the fuzzy

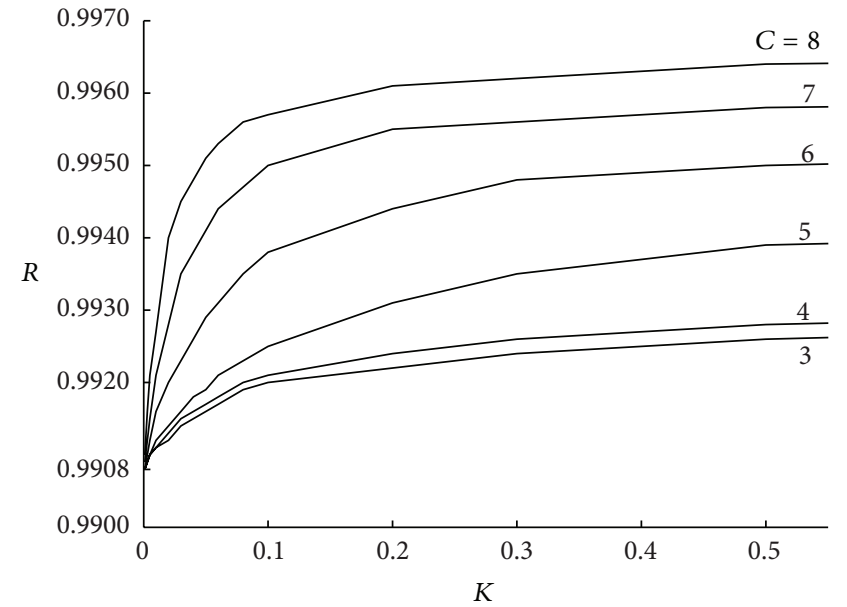

Figure 6: Fuzzy reliabilities with $K$ for different $C$.

degree, the reliability assessments under UD, LD and TND all decrease first and then increase instead, which is another evidence to show the deficiency of UD, LD, and TND on convergence property.

Therefore, the proposed M-TND should have better performance than UD, LD, and TND.

5.2. Regularity of Fuzzy Reliability with the Variation of FuzzyInformation Parameters. In practice, if the engineers only know that the inherent frequency is about $180 \mathrm{~Hz}$ and have no more information, the fuzzy information flank of the flat 


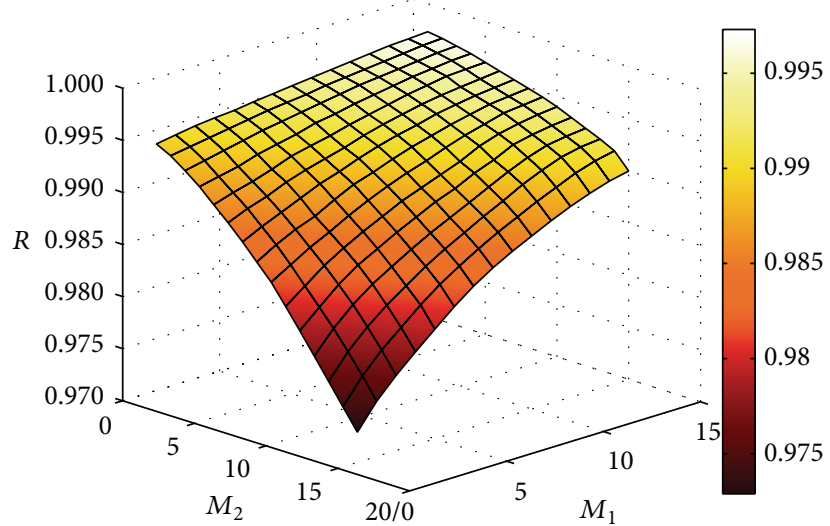

FIgURE 7: Fuzzy reliabilities with $M_{1}$ and $M_{2}$.

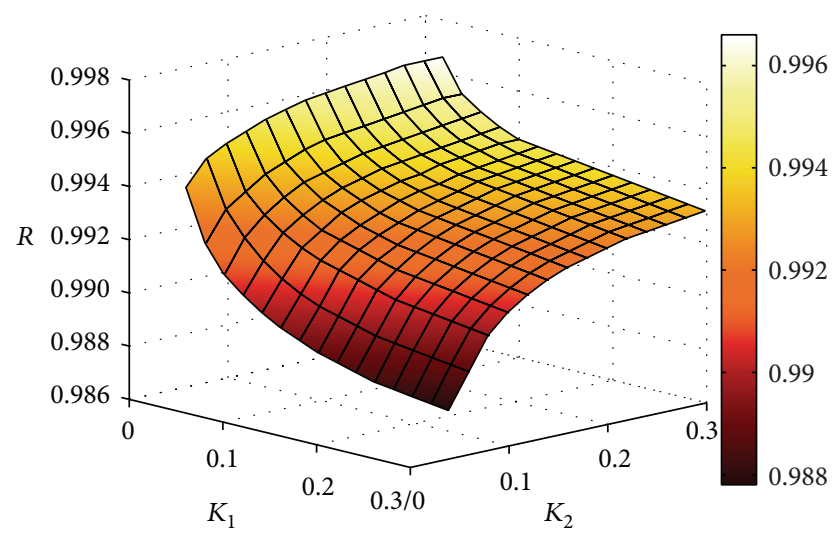

Figure 8: Fuzzy reliabilities with $K_{1}$ and $K_{2}$.

zone is symmetrical; that is, $M_{1}=M_{2}\left(K_{1}=K_{2}\right)$. Conversely, if there is additional information to ascertain that it is more possible that "the inherent frequency is smaller (or bigger) than $180 \mathrm{~Hz}$," the fuzzy information flank is asymmetrical; that is, $M_{1} \neq M_{2}\left(K_{1} \neq K_{2}\right)$. For the symmetrical case, it is shown in Figures 5 and 6 that the higher the fuzzy degree of the inherent frequency is, the smaller the fuzzy reliability will be and vice versa. Thus, numerical simulations will be carried out with the asymmetrical case in Section 5.2.

The variation surface of fuzzy reliabilities with the fuzzyinformation parameters $M_{1}$ and $M_{2}$ is shown in Figure 7 and that with $K_{1}$ and $K_{2}$ is shown in Figure 8. The variation curve families of fuzzy reliabilities with each parameter are shown in Figure 9-12.

It is shown in Figures 7, 9, and 10 that the fuzzy reliability increases with the increasing of $M_{1}$, for given $M_{2}$, and decreases with the increasing of $M_{2}$ for given $M_{1}$. Similarly, Figures 8,11 , and 12 show that the fuzzy reliability decreases with the increasing of $K_{1}$, for given $K_{2}$, and increases with the increasing of $K_{2}$ for given $K_{1}$. In a word, for the given right-side fuzzy information, the fuzzy reliability will increase with the increasing of the fuzzy degree of left-side fuzzy information and vice versa. While, for the given left-side fuzzy information, the fuzzy reliability will decrease with the increasing of the fuzzy degree of right-side fuzzy information

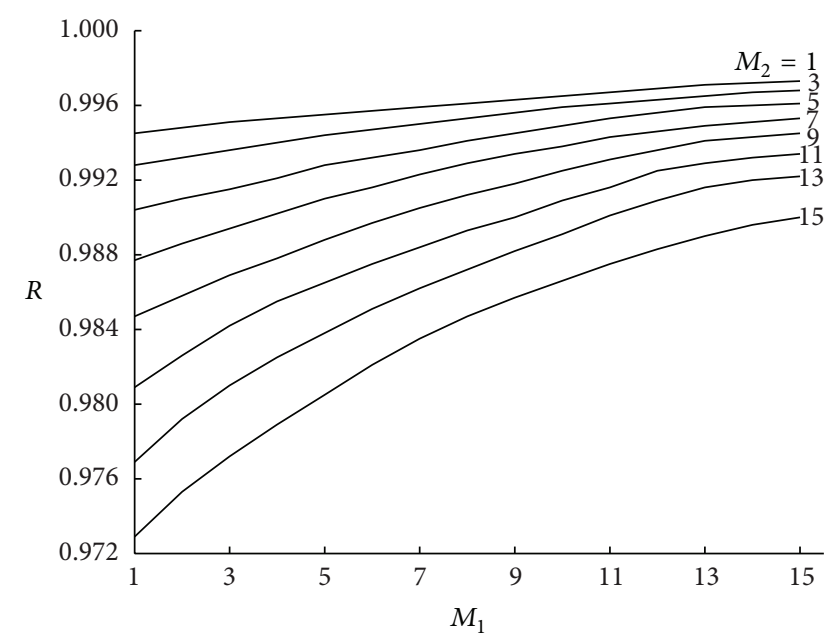

FIGURE 9: Fuzzy reliabilities curve family with $M_{1}$.

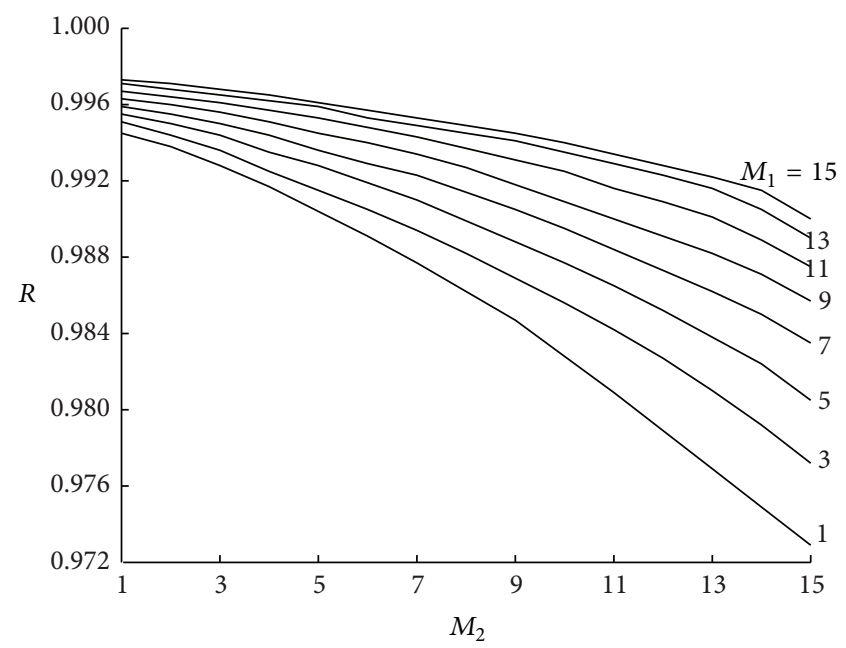

FIgURE 10: Fuzzy reliabilities curve family with $M_{2}$.

and vice versa. This is because of the fact that when the left-side ambiguity increases, the possibility of "the inherent frequency in the left fuzzy zone" will increase. While the mean of the excitation frequency is on right side of the flat zone $[a, b]$, thus, the possibility that "the inherent frequency away from the excitation frequency" increases; that is to say, the reliability increases and vice versa. Similarly, the regularity will be contrary when the mean of the excitation frequency is on the left side of the flat zone.

5.3. Influences of Other Factors on the Fuzzy Reliability. Fuzzy-information parameters and fuzzy degree can only reflect the influence of fuzzy information on reliability. But, besides the fuzzy information, the fuzzy reliability is also related to precise information, that is, the flat zone. Therefore, the conclusions in Section 5.2 are made based on the premise that the flat zone is fixed. Eight experiments are carried out to show the influence of the flat zone on reliability in Table 3 .

It is shown in Table 3 that the change of the position and length of the flat zone will affect the fuzzy reliability even 
TABLE 3: The influence of the flat zone on the fuzzy reliability.

\begin{tabular}{|c|c|c|c|c|c|c|c|c|c|}
\hline \multirow{2}{*}{ Number } & \multicolumn{3}{|c|}{ Precise information } & \multicolumn{3}{|c|}{ Fuzzy information (Trapezoidal) } & \multicolumn{3}{|c|}{ Fuzzy information (Flat-normal) } \\
\hline & Position & Length & Flat zone & $M$ & Fuzzy degree & $R$ & $K$ & Fuzzy degree & $R$ \\
\hline (1) & 180 & 10 & {$[175,185]$} & 5 & 2.5 & 0.9958 & 0.3 & 1.3510 & 0.9963 \\
\hline (2) & 180 & 20 & {$[170,190]$} & 5 & 2.5 & 0.9920 & 0.3 & 1.3510 & 0.9926 \\
\hline (3) & 180 & 10 & {$[175,185]$} & 10 & 5 & 0.9938 & 0.1 & 2.3401 & 0.9956 \\
\hline (4) & 180 & 20 & {$[170,190]$} & 10 & 5 & 0.9915 & 0.1 & 2.3401 & 0.9918 \\
\hline (5) & 185 & 10 & {$[180,190]$} & 5 & 2.5 & 0.9765 & 0.3 & 1.3510 & 0.9780 \\
\hline (6) & 185 & 20 & {$[175,195]$} & 5 & 2.5 & 0.9644 & 0.3 & 1.3510 & 0.9654 \\
\hline (7) & 185 & 10 & {$[180,190]$} & 10 & 5 & 0.9696 & 0.1 & 2.3401 & 0.9755 \\
\hline (8) & 185 & 20 & {$[175,195]$} & 10 & 5 & 0.9634 & 0.1 & 2.3401 & 0.9635 \\
\hline
\end{tabular}

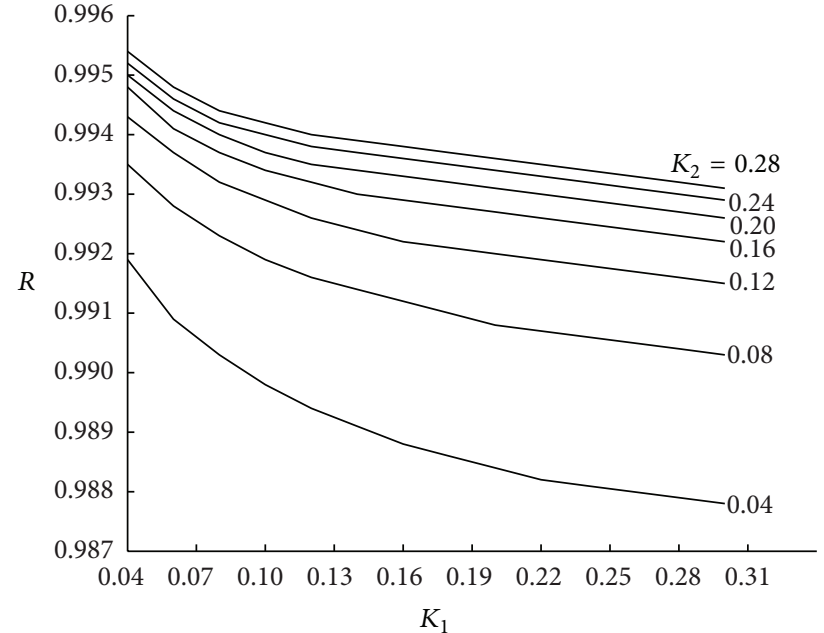

FIGURE 11: Fuzzy reliabilities curve family with $K_{1}$.

for the same fuzzy-information parameters (or fuzzy degree): (1) comparing (2) with (3) (or (6) with (7)), it can be seen that although the fuzzy degree increases, the reliability does not decrease. Instead, it increases due to the shortening of the flat zone. (2) Comparing (3) with (5) (or (4) with (6)), it can be seen that although the fuzzy degree decreases, the reliability does not increase. Instead, it decreases due to the position change of the flat zone.

\section{Conclusions}

The inherent frequencies of engine blades with damping structures often have a lot of fuzziness. In this paper, the inherent frequencies of blades are regarded as fuzzy random variables. The Posbist fuzzy reliability model of blades to avoid resonance is proposed based on the fuzzy cut-set theory. The cut-set distribution is of a significant effect on the Posbist reliability assessment result. A convergence theorem is proposed and proved to show the deficiency of the previous commonly used distributions UD, LD, and TND, and a new distribution M-TND is further presented. Simulation results show that M-TND is of better performance to ensure

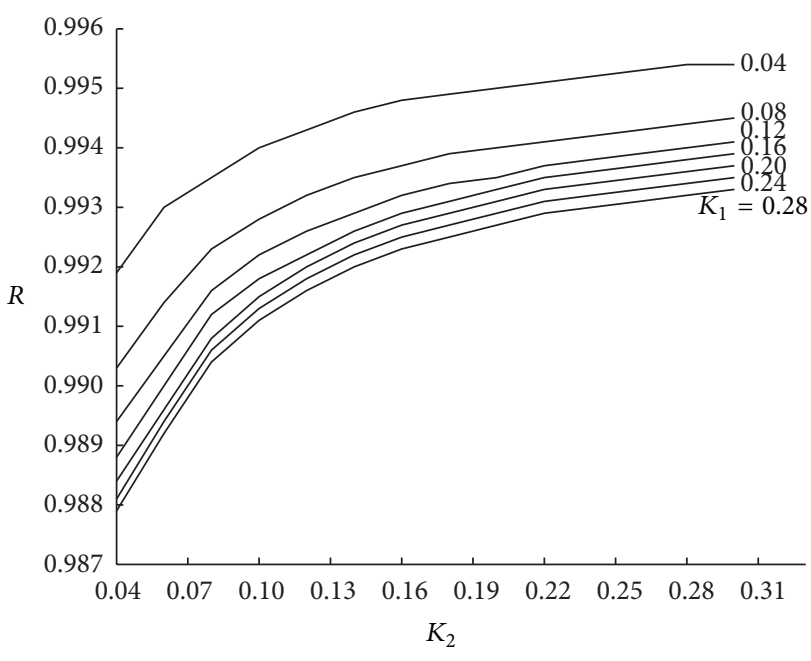

Figure 12: Fuzzy reliabilities curve family with $K_{2}$.

a good convergence of the Posbist model. When the fuzzy information flank is symmetrical, the bigger the fuzzy degree of the inherent frequency is, the smaller the fuzzy reliability will be and vice versa, while when the fuzzy information flank is asymmetrical, the bigger the fuzzy degree on the side near the mean of the excitation frequency is, the smaller the fuzzy reliability will be, and the bigger the fuzzy degree on the side away from the mean of the excitation frequency is, the bigger the fuzzy reliability will be.

\section{Conflict of Interests}

The authors declare that there is no conflict of interests regarding the publication of this paper.

\section{Acknowledgments}

This work has been supported by the National Natural Science Foundation of China (nos. 70471057 and 71171164), and the Natural Science Foundation of the Education Department of Shaanxi Province (no. 03JK065). The authors express their sincere appreciation to Dr. Yang Yang and all the experts 
for their useful comments and help and are very grateful to the reviewers and the editor for their helpful comments and suggestions which have helped them in improving the quality of this paper.

\section{References}

[1] X. Jin, D. Gao, X. Cai, and L. Wu, "Analysis of integrated centrifugal impeller blade vibration reliability," Journal of Aerospace Power, vol. 19, no. 5, pp. 610-613, 2004.

[2] H. Y. Zhang, The Shroud Fan Blade Design for Reducing the Vibration, Chinese Aeronautical Establishment, Beijing, China, 2011.

[3] L. A. Zadeh, "Fuzzy sets," Information and Computation, vol. 8, no. 4, pp. 338-353, 1965.

[4] V. Ravi, P. J. Reddy, and H. Zimmermann, "Fuzzy global optimization of complex system reliability," IEEE Transactions on Fuzzy Systems, vol. 8, no. 3, pp. 241-248, 2000.

[5] F. Biondini, F. Bontempi, and P. G. Malerba, "Fuzzy reliability analysis of concrete structures," Computers and Structures, vol. 82, no. 13-14, pp. 1033-1052, 2004.

[6] H. Wu, "Bayesian system reliability assessment under fuzzy environments," Reliability Engineering and System Safety, vol. 83, no. 3, pp. 277-286, 2004.

[7] S. S. Halilčević, F. Gubina, and A. F. Gubina, "The composite fuzzy reliability index of power systems," Engineering Applications of Artificial Intelligence, vol. 24, no. 6, pp. 1026-1034, 2011.

[8] M. Tanrioven, Q. H. Wu, D. R. Turner, C. Kocatepe, and J. Wang, "A new approach to real-time reliability analysis of transmission system using fuzzy Markov model," International Journal of Electrical Power and Energy System, vol. 26, no. 10, pp. 821-832, 2004.

[9] H. Z. Huang, M. J. Zuo, and Z. Q. Sun, "Bayesian reliability analysis for fuzzy lifetime data," Fuzzy Sets and Systems, vol. 157, no. 12, pp. 1674-1686, 2006.

[10] K. Y. Cai, C. Y. Wen, and M. L. Zhang, "Survivability index for CCNs: a measure of fuzzy reliability," Reliability Engineering and System Safety, vol. 33, no. 1, pp. 71-99, 1991.

[11] K. Y. Cai, C. Y. Wen, and M. L. Zhang, "Fuzzy variables as a basis for a theory of fuzzy reliability in the possibility context," Fuzzy Sets and Systems, vol. 42, no. 2, pp. 145-172, 1991.

[12] K. Y. Cai, C. Y. Wen, and M. L. Zhang, "Posbist reliability behavior of typical systems with two types of failure," Fuzzy Sets and Systems, vol. 43, no. 1, pp. 17-32, 1991.

[13] K. J. Xu, L. P. Jiang, and Y. S. Sui, "Applying fuzzy method to assessment vibration reliability of structural components," Journal of Mechanical Strength, vol. 25, no. 5, pp. 523-526, 2003.

[14] L. Ma, H. X. Zhang, B. Xu et al., "Fuzzy estimation of aeroengine blades operational vibration reliability," Mechanical Science and Technology, vol. 11, no. 25, pp. 1365-1368, 2006.

[15] L. Ma, J. Xie, B. Xu et al., "Study on blade vibration reliability under working environment," Turbine Technology, vol. 49, no. 1, pp. 40-42, 2007.

[16] F. D. Pi, Study on the Structure Vibration Fuzzy Reliability Analysis Method and Its Application, Northwestern Polytechnical University, Xi'an, China, 2006.

[17] J. Sun, Study on the Fuzzy Random Reliability Analysis Methods, Northwestern Polytechnical University, Xian, China, 2005.

[18] H. Z. Huang, "Structural reliability analysis using fuzzy sets theory," Maintenance and Reliability, vol. 14, no. 4, pp. 284-294, 2012.
[19] H. Huang, Z. Sun, D. Guo, L. Li, F. Zhou, and Z. Tian, "Fuzzy reliability analysis in the case of random stress and fuzzy strength," Journal of Mechanical Strength, vol. 23, no. 3, pp. 305307, 2001.

[20] Y. G. Dong, "Fuzzy reliability design with random variable and fuzzy variable," Chinese Journal of Mechanical Engineering, vol. 36, no. 6, pp. 25-29, 2000.

[21] Y. G. Dong, W. Y. Zhu, and X. Z. Chen, "Study on a calculating method of machine fuzzy reliability," Journal of Systems Engineering, vol. 15, no. 1, pp. 7-12, 2000.

[22] Q. M. Jiang and C. H. Chen, "A numerical algorithm of fuzzy reliability," Reliability Engineering and System Safety, vol. 80, no. 3, pp. 299-307, 2003.

[23] Z. Lu, J. Sun, and Y. Xu, "Numerical algorithm for fuzzy reliability analysis of mechanical structure system," Chinese Journal of Mechanical Engineering, vol. 41, no. 9, pp. 19-23, 2005.

[24] S. L. Chen, J. G. Li, and X. G. Wang, Fuzzy Set Theory and Application, Science Press, Beijing, China, 1st edition, 2005.

[25] H. He and Z. Lu, "Reliability analysis method for structure with fuzzy variable," Journal of Mechanical Strength, vol. 31, no. 4, pp. 609-614, 2009. 


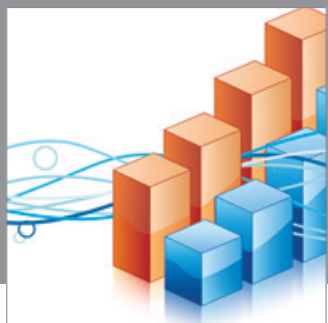

Advances in

Operations Research

mansans

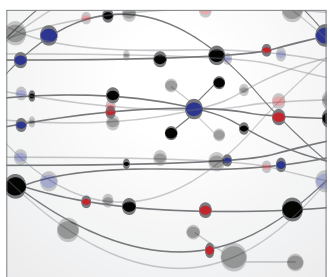

The Scientific World Journal
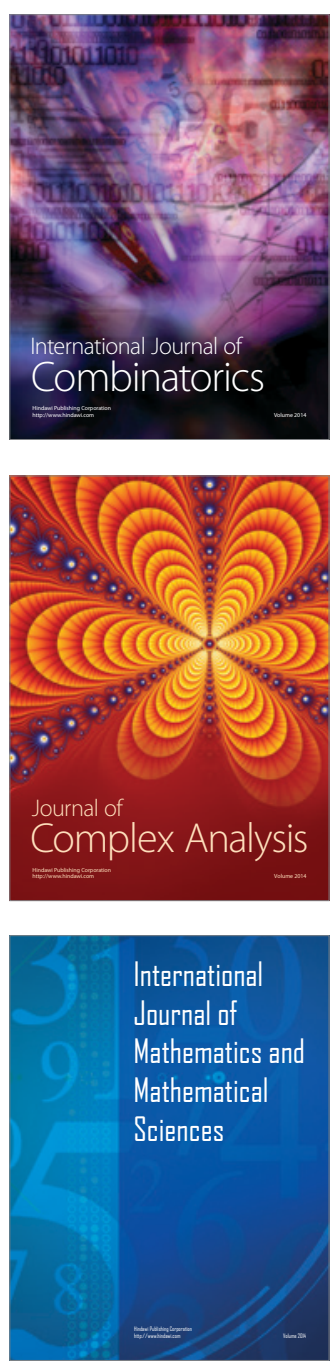
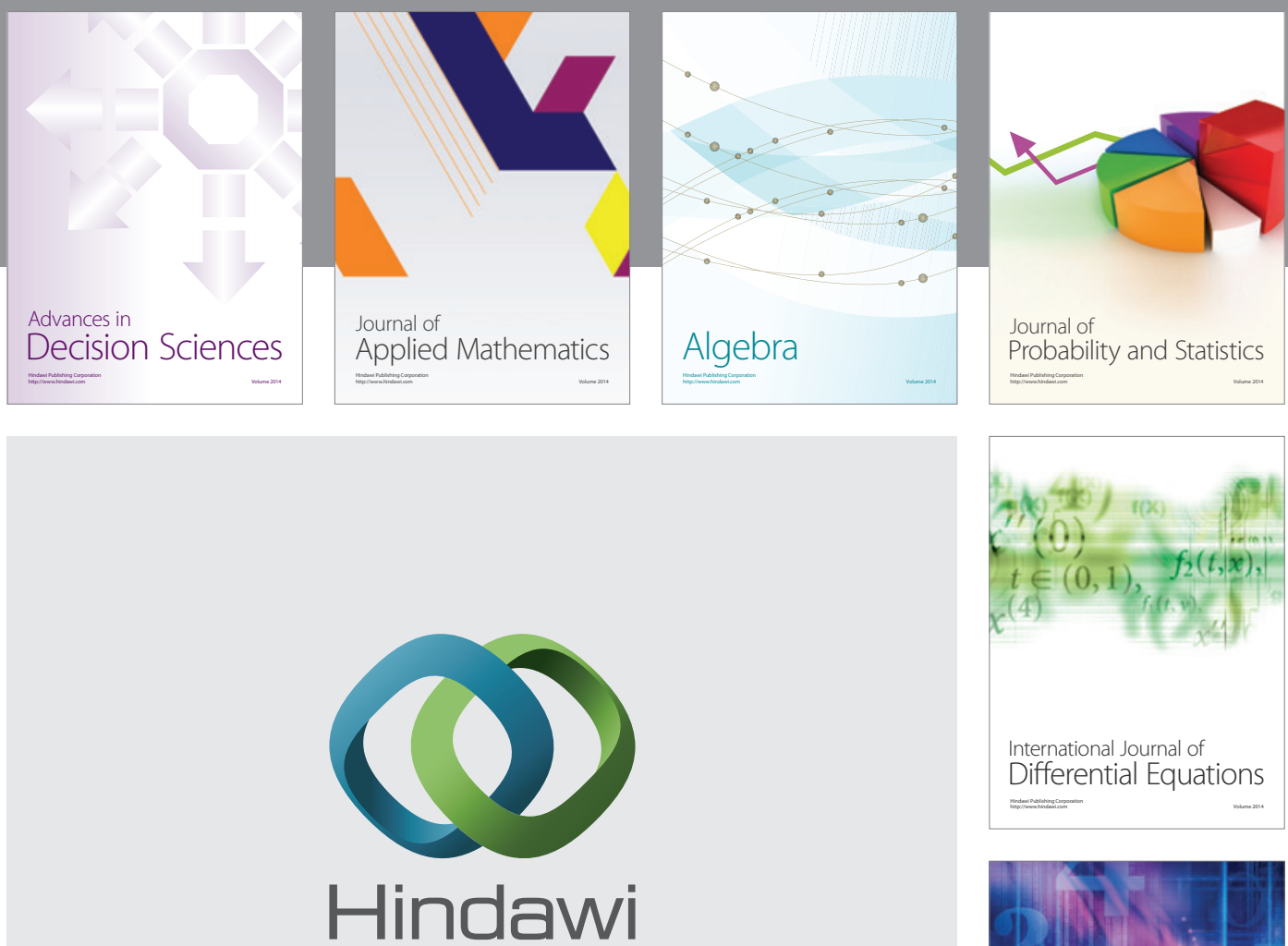

Submit your manuscripts at http://www.hindawi.com
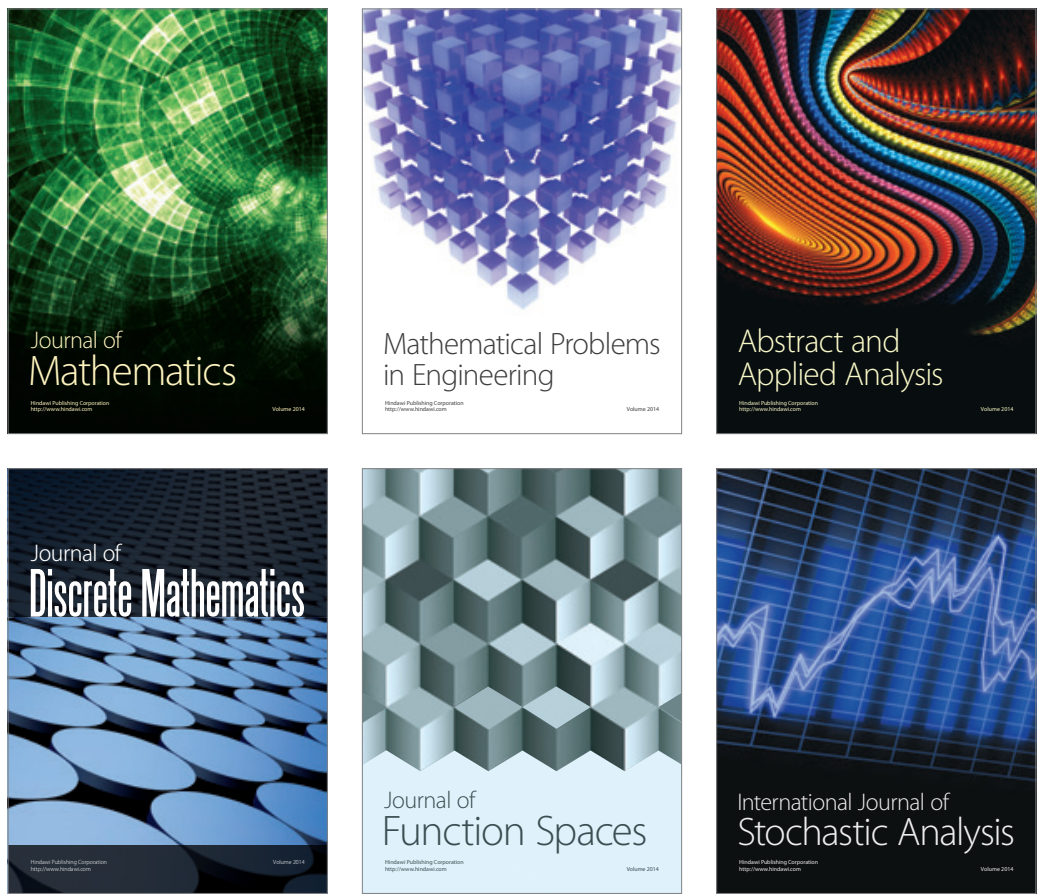

Journal of

Function Spaces

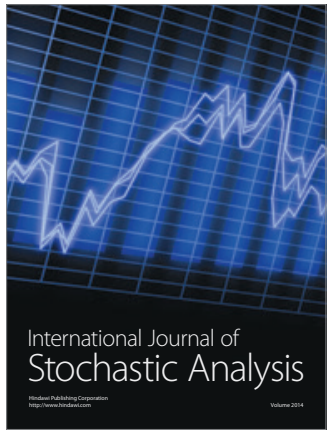

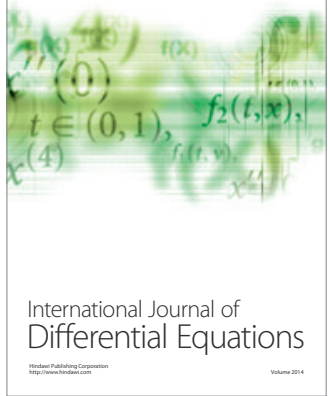
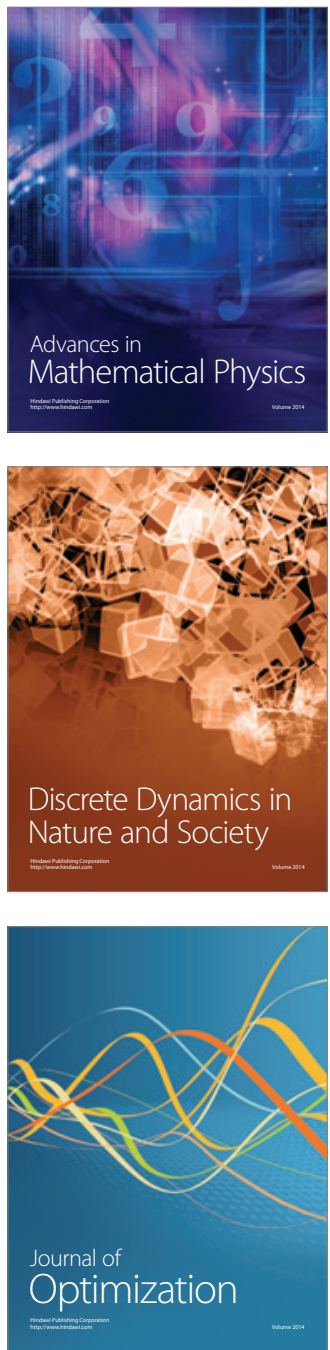